\title{
Multi-objective Environmental-economic Load Dispatch Considering Generator Constraints and Wind Power Using Improved Multi-objective Particle Swarm Optimization
}

\author{
Tankut YALCINOZ ${ }^{1,2}$ Krzysztof RUDION $^{1}$ \\ ${ }^{1}$ IEH, University of Stuttgart, Stuttgart, Germany \\ ${ }^{2}$ TransnetBW GmbH, Stuttgart, Germany \\ tyalcinoz@ieee.org
}

\begin{abstract}
One of the vital optimization issues in energy systems is the problem of economic load dispatch (ED). On the other hand, solar, wind, and other renewable energies are important energy sources for reducing hazardous emissions. This paper suggests an improved multi-objective particle swarm optimization algorithm (IMOPSO) that uses a functional inertial weight and a functional constriction factor to solve the multi-objective environmental-economic load dispatch (MEED) problem. A mutation strategy is used in IMOPSO, and a mutation operator, which is implemented for each particle in the swarm, is used to find optimum Pareto fronts. In this paper, the proposed IMOPSO is applied to the MEED problem under consideration of emission pollution, wind energy, prohibited operating zone, ramp limits, valve point effects, and transmission losses. The proposed technique is tested on the IEEE 30-bus, the IEEE 118-bus test system, and the modified IEEE 118-bus test system with emission coefficients, ramp rate limits, wind power, and prohibited operating zone. The IMOPSOs are compared with the results of various multi-objective algorithms to solve the MEED problem. The simulation results indicate that the IMOPSO produces better results than the compared multi-objective optimization algorithms for various test systems.
\end{abstract}

Index Terms - optimization, particle swarm optimization, power generation dispatch, power system economics, wind energy.

\section{INTRODUCTION}

Optimization has always been used for solving energy system problems to achieve maximum benefit at minimum cost. In the planning and operation of energy systems, economic load dispatch (ED) is one of the crucial optimization issues seeking to define the optimal outputs of generation units to reduce overall generation costs while satisfying various equality and inequality constraints.

In recent years, numerous optimization techniques have been suggested to solve various ED problems [1-27]. The traditional numerical approaches such as linear programming [1], Lagrangian multiplier method [2], lambda-iteration method [2], Newton method [2], interior point method [3], and quadratic programming algorithm [4] were applied in solving the ED problems. However, the application of traditional numerical methods to nonlinear economic dispatch problems becomes more complicated.

Various metaheuristic optimization techniques have been suggested in the literature for solving nonlinear ED problems [5-26]. The application of metaheuristic optimization methods such as genetic algorithm [5], evolutionary programming [6], differential evolution [7], crisscross optimization [8], biogeography-based optimization (BBO) [9], particle swarm optimization (PSO) [10-13], Flower pollination algorithm [14], ant colony optimization [15], artificial immune system [16] and bacterial foraging optimization (BFO) [17] showed promising solutions for different ED problems considering various restrictions such as transmission losses and generator constraints. The cost functions, even if they are non-smooth, non-convex, and non-linear functions, can be easily solved by metaheuristic optimization techniques. Besides, metaheuristic optimization techniques can produce a global or near-global solution. However, some metaheuristic approaches face early convergence, and a long computing time as the size of the system increases. Abbas et al [11] reported that PSO has many remarkable characteristics, such as ease of implementation, flexibility, less storage space, the capability of finding high-quality solutions with less computing time, and significant convergence features compared to alternative metaheuristic optimization techniques.

Multi-objective optimization problems consist of many objective functions that need to be addressed simultaneously. A multi-objective optimization problem can have many Pareto-optimal solutions. The task of a multiobjective optimizer is to find the optimal solution correctly. Several multi-objective optimization methods and applications have been reported in the literature [1], [6], [14] and [18-26].

Increasing environmental concerns in recent years have made it imperative to reduce not only fuel costs but also pollutant emissions [5-6]. The multi-objective environmental-economic load dispatch problem, which is one of the constrained nonlinear multi-objective optimization problems, consists of two conflicting objective functions. Numerous methods for solving the economic dispatch problem with a multi-objective function have been proposed [5], [6], [8], [11-14] and [18-26]. Qu et al [6] reported the application of multi-objective evolutionary algorithms to solve the MEED and economic dispatch problems. A multi-objective global best artificial bee colony 
algorithm (MOGABC) is presented to solve the MEED problem [18]. A hybrid multi-objective optimization algorithm based on differential evolution and particle swarm optimization (MODE/PSO) is employed on the MEED problem [19]. A multi-objective stochastic search technique (MOSST) is proposed as a hybrid of a real-coded genetic algorithm and simulated annealing techniques for economic load dispatch problem [20]. Abido proposed a niched Pareto genetic algorithm (NPGA) [21], a strength Pareto evolutionary algorithm (SPEA) [22], and a non-dominated sorting genetic algorithm (NSGA) [24] for the MEED problem. A fuzzy clustering-based Particle Swarm (FCPSO) algorithm, which handles the size of the repository within limits without destroying the Pareto frontal features, is suggested to address the MEED problem [23]. A nondominated sorting genetic algorithm-III (NSGA-III), which can handle non-linear problems, is proposed to solve the MEED problem [25]. Sivasubramani and Swarup [26] presented a multi-objective harmony search algorithm and a fast non-dominated sorting genetic algorithm (NSGA-II) method for the MEED problem.

Recently, many problems of energy systems have been successfully solved by the particle swarm optimization approach [10-13]. Particle Swarm Optimization (PSO), which is an effective metaheuristic optimization method, stimulates the social behavior of the animals [27]. For many challenging optimization problems, it performs better than the other optimization methods at quicker and more robust rates of convergence [28-40]. Bonyadi and Michalewicz [28] reported a comprehensive study of limitations, modifications and applications of PSO types for optimization problems. However, the stability and convergence of the PSO algorithm were investigated by Clerc and Kennedy [29]; and the constriction factor was implemented to guarantee the convergence of the PSO algorithm [29]. In order to generate the diversity of the population in PSO, a mutation operator was proposed [30]. A new strategy for fitness estimation in the PSO algorithm is proposed to reduce the computing time [31]. Different strategies for inertial weights were described, and a new adaptive approach for inertial weights was proposed by Nickabadi et al [32]. Lu et al [33] suggested two enhanced PSO versions that employ both functional inertia weight and functional constriction factor. When selecting the text attributes, it was demonstrated that the asynchronous improved PSO approach yielded the best outcomes among the compared methods. In this paper, we examine a multiobjective particle swarm optimization using an asynchronous inertia weight and the constriction factor in order to enhance the performance of solving power optimization problems.

In this paper, the approach based on a multi-objective particle swarm optimization for solving multi-objective environmental-economic load dispatch problem with thermal and wind power generators is proposed. The different test systems have been employed to demonstrate the potential attributes of the proposed algorithm. The first test system is the IEEE 30-bus six-unit test system with emission coefficients. The second test system considers the IEEE 118-bus test system that contains 118-Buses and 14 generators with emission coefficients. The third test system is the IEEE 118 bus test system with emission coefficients, ramp rate limits, and prohibited operating zone. Finally, the last test system is the modified IEEE 118-bus test system with emission coefficients, ramp rate limits, wind power, and prohibited operating zone. The simulation results of the proposed IMOPSO are evaluated against those of MOGABC [18], MODE/PSO [19], LP [1], MOSST [20], NPGA [21], SPEA [22], FCPSO [23], NSGA [24], NSGAIII [25] and NSGA-II [26].

\section{ECONOMIC AND EMISSION DiSPATCH PROBLEM FORMULATION}

The economic dispatch problem is to reduce fuel costs while meeting the load demand and many equality and inequality constraints [2]. In this paper, the multi-objective environmental-economic load dispatch problem consists of three objective functions that are the fuel cost, wind power cost, and emission functions. The multi-objective environmental-economic load dispatch problem is an expansion of the economic/emission dispatch problem by considering emission pollution, wind energy, prohibited operating zone, ramp limits, valve point effects, and transmission losses.

As a multi-objective optimization problem, the MEED problem can be described as follows

$$
\operatorname{Min}\left[F_{C, t}, F_{w, t}, F_{E, t}\right]
$$

Subject to

$$
\begin{aligned}
& \text { Equality constraints } g\left(P, P_{w}\right)=0 \\
& \text { Inequality constraints } \quad h\left(P, P_{w}\right) \leq 0 \\
& P_{i, \min } \leq P_{i} \leq P_{i, \max }
\end{aligned}
$$

where $\mathrm{P}$ and $\mathrm{P}_{\mathrm{w}}$ are variables associated with power outputs of conventional generator units and wind turbines.

- Minimization of fuel cost:

In classical ED problems, the fuel cost function of the conventional generator unit can be specified as a secondorder function. The total generation $\operatorname{cost} \mathrm{F}_{\mathrm{C}, \mathrm{t}}$ of the conventional generator units is written as

$$
\text { Min } F_{C, t}=\sum_{i=1}^{n}\left(a_{i}+b_{i} P_{i}+c_{i} P_{i}^{2}\right)
$$

On the other hand, if the valve point loading effects are considered in the fuel cost objective function, the ED problem turns into a non-linear and non-smooth problem. In the case of the fuel cost function including the valve point load effects, the objective function is represented in the following equation

$$
\text { Min } F_{C, t}=\sum_{i=1}^{n}\left(\begin{array}{l}
a_{i}+b_{i} P_{i}+c_{i} P_{i}^{2}+ \\
\left|d_{i} \sin \left(e_{i}\left(P_{i, \min }-P_{i}\right)\right)\right|
\end{array}\right)
$$

where $a_{i}, b_{i}$, and $c_{i}$ are the cost coefficients of the i-th unit, $d_{i}$ and $e_{i}$ are constants that represent valve point effects, and $n$ is the number of conventional generators, respectively.

- Minimization of the wind power cost:

The cost function may not be present where the system operator owns the wind turbines since the wind power do not require fuel [41]. However, if a wind turbine is not owned by a utility, the cost of wind power generation must be determined based on the specific contracts [42]. In this paper, the total cost function $\mathrm{F}_{\mathrm{w}, \mathrm{t}}$ of wind power generators 
can be represented as a linear function [42]

$$
\text { Min } \quad F_{w, t}=\sum_{i=1}^{n w} g_{i} P_{w i}
$$

where $P_{w i}$ is the power output of the ith wind generator, nw is the number of wind generators and $g_{i}$ is the cost coefficient of the ith wind generator.

- Minimization of the emission:

The total environmental emission $\mathrm{F}_{\mathrm{E}, \mathrm{t}}$ (ton/h) of air pollutants such as sulfur oxides SOx and nitrogen oxides NOx produced by thermal units are modeled as follows:

$$
\text { Min } F_{E, t}=\sum_{t=i}^{n}\left(\begin{array}{l}
10^{-2}\left(\alpha_{i}+\beta_{i} P_{i}+\gamma_{i} P_{i}^{2}\right)+ \\
\zeta_{i} \exp \left(\lambda_{i} P_{i}\right)
\end{array}\right)
$$

where $\alpha_{i}, \beta_{i}, \gamma_{i}, \zeta_{i}$ and $\lambda_{i}$ are the emission coefficients of the i-th generation unit respectively.

- Problem constraints:

In solving the MEED problem, several equality and inequality constraints of generators and systems must be met. In this paper, the MEED problem is subject to the subsequent constraints:

- Power balance equation:

The sum of generated power should meet total demand $\mathrm{P}_{\mathrm{D}}$ and transmission losses $\mathrm{P}_{\mathrm{L}}$, expressed as follows

$$
\sum_{i=1}^{n} P_{i}+\sum_{i=1}^{n w} P_{w i}-P_{D}-P_{L}=0
$$

The transmission losses are calculated by the $\mathrm{B}$ matrix loss method, which is described as

$$
P_{L}=\sum_{i=1}^{n} \sum_{j=1}^{n} P_{i} B_{i j} P_{j}+\sum_{i=1}^{n} B_{o i} P_{i}+B_{00}
$$

where $\mathrm{B}_{\mathrm{ij}}, \mathrm{B}_{0 \mathrm{i}}$ and $\mathrm{B}_{00}$ represent coefficients of transmission losses, respectively.

- Power limits of units

Each unit must be operated according to the minimum $P_{i, \min }$ and maximum $P_{i, \max }$ generation capacities as follows

$$
P_{i, \text { min }} \leq P_{i} \leq P_{i, \max }, \text { for } i=1, \ldots, n
$$

- The power outputs of wind generators

The power output $P_{w i}$ of the i-th wind generator fluctuates depending on the wind speed $\left(v_{i}\right)$ and is represented as follows [42-43]

$$
P_{w i}(v)=\left\{\begin{array}{ccc}
0 & \text { for } \quad v_{i}\left\langle v_{i, \text { in }} \text { and } v_{i}\right\rangle v_{i, \text { out }} \\
P_{i, w r} & \left(\frac{v_{i}-v_{i, \text { in }}}{v_{r i}-v_{i, \text { in }}}\right) \quad \text { for } v_{i, \text { in }} \leq v_{i} \leq v_{r i} \\
& P_{i, w r} \text { for } \quad v_{r i}\left\langlev \left\langle v_{i, \text { out }}\right.\right.
\end{array}\right\}
$$

where, $P_{i, w r}$ is the rated power output of the $\mathrm{i}$-th wind generator. $v_{r i}, v_{i, \text { in }}$ and $v_{i, \text { out }}$ are the rated, cut-in and cut-out wind speeds of the $\mathrm{i}$-th wind generator, respectively.

- $\quad$ Ramp rate limits

Their ramp-up and ramp-down rates limit the operating range of generation units. Therefore, the operating limits of real power are specified as follows:

$$
\max \left(P_{i, \min }, P_{i}^{0}-D R_{i}\right) \leq P_{i} \leq \min \left(P_{i, \max }, P_{i}^{0}+U R_{i}\right)
$$

where $P_{i}^{0}$ is the initial power output of the $\mathrm{i}$-th unit. $\mathrm{UR}_{\mathrm{i}}$ and $\mathrm{DR}_{\mathrm{i}}$ are the ramp-up limits and ramp-down limits of the i-th generation unit, respectively.

- $\quad$ Prohibited operation zone

In practice, the units may have specific operating ranges for load sharing based on the physical operating limits of the machine components. Therefore, units with prohibited operation zones have discontinuous fuel-cost characteristics. The feasible power generation limits of units with prohibited operation zones are determined as follows:

$$
P_{i} \in\left\{\begin{array}{c}
P_{i, \min } \leq P_{i} \leq P_{i, L 1} \\
P_{i, U z-1} \leq P_{i} \leq P_{i, L z}, z=2,3, \ldots, n p z \\
P_{i, U z} \leq P_{i} \leq P_{i, \max }
\end{array}\right.
$$

where $P_{i, U z}$ and $P_{i, L z}$ are lower and upper power limits of the prohibited operation zones of the i-th generation unit, respectively.

\section{Multi-ObJective Particle Swarm Optimization}

Many multi-objective particle swarm optimization (MOPSO) algorithms have been proposed to solve the problem of multi-objective optimization in the literature [13] and [37-40]. Many MOPSOs algorithms concentrate on procedures for choosing the best global, and some MOPSOs use a fixed value for dynamics and accelerations. Recently, various modifications have been implemented to the typical PSO in order to achieve the optimum solution more quickly [28-36]. In this paper, the typical PSO algorithm is improved by defining the functional inertia weight and the functional constriction factor to improve the overall PSO performance. Moreover, the improved particle swarm optimization algorithm is extended for solving multiobjective economic dispatch problems, which consists of several objectives and many equality and inequality constraints. In IMOPSO, the mutation operator is employed to get convergence into a Pareto front [37].

Particle Swarm Optimization uses a set of particles that pass through the search space to find the global minimum. In PSO, each particle offers a possible solution for the problem to be solved. In PSO, each particle $\mathrm{i}$ is related to the velocity $\mathrm{v}_{\mathrm{i}}$ and the position $\mathrm{x}_{\mathrm{i}}$. Firstly, the velocity and position of each particle are set randomly. Then the velocity and position of the $\mathrm{i}$-th particle are updated as follows

$$
\begin{aligned}
& v_{i}(t+1)=v_{i}(t)+c_{1} r_{1}\left(\text { pbest }_{i}(t)-x_{i}(t)\right)+ \\
& c_{2} r_{2}\left(\text { gbest }_{i}(t)-x_{i}(t)\right) \\
& x_{i}(t+1)=x_{i}(t)+v_{i}(t+1)
\end{aligned}
$$

where pbest $t_{i}$ is the best position of the $\mathrm{i}$-th particle; gbest $_{i}$ is the best position of the full swarm; $t$ is the iteration number; the personal $c_{1}$ and global learning $c_{2}$ coefficients are generally assumed to be 2.0. The uniformly distributed random values $\left(r_{1}\right.$ and $r_{2}$ ) have a range of $[0,1]$.

Equation (15) consists of the inertia weight term, the cognitive term representing the personal effect of the particle, and the social part representing the social effect of the particle. Many variations of the PSO algorithm have been proposed to solve problems of constrained optimizations [28-36]. Abido [34] suggested a dynamic value that can be allocated to the maximum velocity. This study defines the velocity limits as follows

$$
-\frac{x_{\max }-x_{\min }}{c_{\text {int }}} \leq x_{i} \leq \frac{x_{\max }-x_{\min }}{c_{\text {int }}}
$$

where $\mathrm{x}_{\max }$ and $\mathrm{x}_{\min }$ are the limits of particles in the swarm and $\mathrm{c}_{\mathrm{int}}$ is the coefficient in the range $\left[1, \mathrm{t}_{\mathrm{max}}\right]$. 
It was proposed to apply the constriction factor to improve the overall efficiency of the PSO [29], [35-36]. A constriction factor for the PSO algorithm was proposed for the solution of the economic load dispatch, including valve point loading effects [36]. The constriction factor was proposed to achieve the best convergence of the PSO algorithm by Clerc and Kennedy [29]. Lu et al [33] showed that the constriction factor chosen as a convex function prevented early convergence in early iterations. In addition, two modified PSO types were reported, which use both the functional constriction factor and the functional inertial weight [33]. In this study, an asynchronous inertia weight and constriction factor in PSO are employed, as defined by $\mathrm{Lu}$ et al [33]. The structure of the constriction factor based on the cosine function can be formulated as follows [33]

$$
\kappa=\frac{\cos \left(2 \frac{\pi}{t_{\max }}\left(t-t_{\text {max }}\right)+2,428571\right)}{4}
$$

where $t_{\max }$ is the maximum iteration number.

The improved particle swarm optimization (IPSO) is employed to solve economic load dispatch problems [10]. However, The IPSO cannot find Pareto optimal solutions for the MEED problem. Some modifications have been implemented in the PSO to address a multi-objective optimization problem [37-40]. There is typically a contradiction between the objective functions, and consequently, there are a number of solutions determined by the Pareto optimality theory [37-38]. Although each particle in the swarm can contain multiple leaders, only one of the leaders can be chosen to update the velocity in MOPSO. Furthermore, the leaders must ensure diversity in the search to eliminate approaching a single solution [37-38].

The velocity and position of the i-th particle are modified in the following equations

$$
\begin{gathered}
v_{i}(t+1)=\kappa\left(\begin{array}{l}
w v_{i}(t)+c_{1} r_{1}\left(\text { pbest }_{i}(t)-x_{i}(t)\right) \\
+c_{2} r_{2}\left(\text { lbest }_{i}(t)-x_{i}(t)\right)
\end{array}\right) \\
x_{i}(t+1)=x_{i}(t)+v_{i}(t+1)
\end{gathered}
$$

where lbest is the leader of the i-th particle. The personal learning coefficient $c_{1}$ and the global learning coefficient $c_{2}$ are normally assumed to be 2.0. In this study, $\mathrm{c}_{1}$ and $\mathrm{c}_{2}$ are calculated again in each iteration $t$, expressed as

$$
\begin{aligned}
& c_{1}=2+\frac{t_{\text {max }}+t}{5 t_{\text {max }}} \\
& c_{2}=0.4+\frac{t_{\text {max }}+3 t}{2 t_{\text {max }}}
\end{aligned}
$$

In the beginning, particles with a high personal learning coefficient $c_{1}$ and a small global learning coefficient $c_{2}$ are permitted to move in the search space by varying the learning coefficients with iterations. However, for the convergence of the particles to the global optima in the continuing part of the optimization, a small personal learning coefficient, and a high global learning coefficient are produced.

For the stability and convergence behavior of PSOs, the inertial weight factor $\mathrm{w}$ is an important component. Some researchers calculated the inertial weight factor by using an inertial weight-damping ratio and a linear adaptive formulation [31]. The PSO performance is considerably improved by a linear decreasing inertia weight factor. This study updates the inertial weight factor $w$ in each iteration $t$ as shown below [31]

$$
w=w_{\max }-\frac{w_{\max }-w_{\min }}{t_{\max }} t
$$

where $w_{\min }$ and $w_{\max }$ are typically specified as 0.4 and 0.95 , respectively.

The application of mutation operators could considerably enhance the overall efficiency of MOPSO techniques as discussed in the literature [37-38]. Coello et al [37] proposed a mutation operator, which is applied both to the particles of the swarm and to the set of each design variable of the problem under examination. In this paper, the mutation operator, which is designed as a non-linear function, is used for convergence to an optimum Pareto front as reported by Coello et al [37]. In this paper, the influence of the mutation operator to the particles decreases with increasing the iteration number.

In the proposed IMOPSO method, the improved particle swarm optimization is extended to solve the MEED problem, and Pareto optimum solutions are obtained by using the multi-objective particle swarm optimization [3738]. An external archive for storing the non-dominated particles is constructed in IMOPSO as defined in the literature [37-38]. The IMOPSO algorithms use a nondominant classification strategy and different quality scales that are suggested for each particle in the swarm to determine a leader. The results are stored in the external archive and the dominated ones are discarded from the external archive to keep the size of the archive [37-38].

\section{A. Implementation of IMOPSO on MEED}

The improved multi-objective particle swarm optimization is applied to solve different economic load dispatch problems using Matlab. The process steps of IMOPSO for solving various economic load dispatch problems are given in the following:

1. Read data; coefficients of cost and emission, total demand, loss coefficients, other limits, and coefficients.

\section{Define ED problem or MEED problem}

3. Set PSO parameters; the maximum number of iteration, swarm size, lower and upper limits of variables, number of decision variables, size of decision variables matrix, size of the external archive, constriction coefficients, inertia weight, mutation rate.

4. Calculate the constriction factor, personal learning coefficient, and global learning coefficient using equations (18), (21) and (22) respectively.

5. Determine the velocity limits using (17).

6. Determine the actual power operating limits dependent on their ramp-up and ramp-down rates of generation units using (13).

7. Determine the power outputs of the wind generators, which fluctuate depending on the wind speed using (12).

8. Initialize the velocity and position of the individual particles at random; evaluate each of the particles in the swarm; update individual best; define dominance.

9. Determine the leader.

10. Update the velocity and position of the individual 
particle using equations (19) and (20), respectively.

11. Apply velocity limits and position limits.

12. Calculate each of the particles in the swarm; determine the power outputs of all units; calculate the cost function, the wind power cost function, and the emission function; calculate transmission loss; calculate power balance violation.

13. Apply the mutation described by Coello et al [37].

14. Update personal best.

15. Update dominance

16. Update the personal learning coefficient $\mathrm{c}_{1}$ and the global learning coefficient $\mathrm{c}_{2}$ using equations (21) and (22).

17. Update the inertia weight factor using (23).

18. Repeat steps 9-18 up to the maximum number of iterations.

19. Identify the best compromise solution

The power output vector of the units is expressed as the position of the particle in the swarm. Each particle in the swarm is calculated; Unit power outputs are calculated considering ramping rates, valve-point loading effects, transmission losses, and prohibited operating zones.

\section{Simulation Results}

In this paper, the performance of IMOPSO in solving the multi-objective environmental-economic load dispatch problem is investigated for four different test systems. The parameters of IMOPSO were defined as follows after a series of tests:

- the maximum number of iterations is 2000 ,

- the swarm size is 150 ,

- $\quad$ the mutation rate is 0.1 ,

- boundaries of particles in the swarm are $x_{\max }=1$ and $\mathrm{x}_{\min }=0$.

\section{A. Case Study 1}

In the first case study, the IEEE 30-bus six-unit system with emission coefficients is tested. The system data, including fuel cost coefficients and emission coefficients and B-coefficients, are given in the literature [18-19]. In this study, a total of 2.834 p.u demand based on 100 MVA is determined. We compare the proposed IMOPSO with other different multi-objective algorithms, including MOGABC [18], MODE/PSO [19], LP [1], MOSST [20], NPGA [21], SPEA [22], and FCPSO [23] for the economic dispatch, emission dispatch, and the MEED.

For the economic dispatch, Table I shows simulation results of IMOPSO, MOGABC [18], MODE/PSO [19], LP [1], MOSST [20], NPGA [21], SPEA [22], and FCPSO [23]. As can be seen from Table I, the fuel costs of IMOPSO and MOGABC [18] are lower than those of other multiobjective algorithms, and the proposed algorithm produces slightly lower emissions than MOGABC [18].

For the emission dispatch, Table I demonstrates simulation results of MOGABC [18], MODE/PSO [19], LP [1], MOSST [20], NPGA [21], SPEA [22], and FCPSO [23]. In Table I, IMOPSO, MOGABC [18] and MODE/PSO [19] produce the same emission of $0.194203 \mathrm{ton} / \mathrm{h}$. However, the fuel cost of IMOPSO is $637.9562 \$ / \mathrm{h}$, which is minimum compared to other multi-objective algorithms.

For the best compromise solution for MEED, Table II demonstrates simulation results of IMOPSO, MOGABC
[18], NPGA [21], SPEA [22] and NSGA [24].

TABLE I. RESUlts OF ECONOMIC DiSPATCH AND EMISSION DisPATCH

\begin{tabular}{ccccc}
\multicolumn{2}{c}{ TABLE I. RESULTS OF ECONOMIC DISPATCH AND EMISSION DISPATCH } \\
\hline Methods & $\begin{array}{c}\text { Economic dispatch } \\
\text { Fuel cost } \\
\mathbf{( \$ / h )}\end{array}$ & $\begin{array}{c}\text { Emission } \\
\mathbf{( t o n} / \mathbf{h})\end{array}$ & $\begin{array}{c}\text { Emission } \\
\text { Fuel cost } \\
\mathbf{( \$ / h )}\end{array}$ & $\begin{array}{c}\text { dispatch } \\
\text { Emission } \\
\mathbf{( t o n} / \mathbf{h})\end{array}$ \\
\hline IMOPSO & 600.1114 & 0.222068 & 637.9562 & 0.194203 \\
MOGABC [18] & 600.1114 & 0.22211 & 638.253 & 0.194203 \\
MODE/PSO[19] & 600.115 & 0.22201 & 638.27 & 0.194203 \\
LP[1] & 606.314 & 0.2233 & 639.6 & 0.194227 \\
MOSST [20] & 605.889 & 0.2222 & 644.112 & 0.194182 \\
NPGA [21] & 600.259 & 0.22116 & 639.18 & 0.194327 \\
SPEA [22] & 600.15 & 0.22151 & 638.507 & 0.19421 \\
FCPSO [23] & 600.132 & 0.22226 & 638.358 & 0.194207 \\
\hline
\end{tabular}

TABLE II. RESUlTS OF BEST COMPROMISE SOLUTION FOR MEED

\begin{tabular}{cccccc}
\hline $\begin{array}{c}\text { Unit } \\
\text { (MW) }\end{array}$ & IMOPSO & $\begin{array}{c}\text { MOGABC } \\
{[\mathbf{1 8}]}\end{array}$ & $\begin{array}{c}\text { NSGA } \\
{[\mathbf{2 4}]}\end{array}$ & $\begin{array}{c}\text { NPGA } \\
{[\mathbf{2 1}]}\end{array}$ & $\begin{array}{c}\text { SPEA } \\
{[\mathbf{2 2}]}\end{array}$ \\
\hline P1 & 0.2751 & 0.24 & 0.2571 & 0.2696 & 0.2785 \\
P2 & 0.3875 & 0.3628 & 0.3774 & 0.3673 & 0.3764 \\
P3 & 0.4965 & 0.5425 & 0.5381 & 0.5594 & 0.53 \\
P4 & 0.7661 & 0.7172 & 0.6872 & 0.6496 & 0.6931 \\
P5 & 0.4893 & 0.5416 & 0.5404 & 0.5396 & 0.5406 \\
P6 & 0.4195 & 0.4299 & 0.4337 & 0.4486 & 0.4153 \\
\hline $\begin{array}{c}\text { Fuel cost } \\
\text { (\$/h) }\end{array}$ & 607.960 & 608.1665 & 610.067 & 612.127 & 610.254 \\
$\begin{array}{c}\text { Emission } \\
\text { (ton/h) }\end{array}$ & 0.202 & 0.202 & 0.2006 & 0.19941 & 0.20055 \\
\hline
\end{tabular}

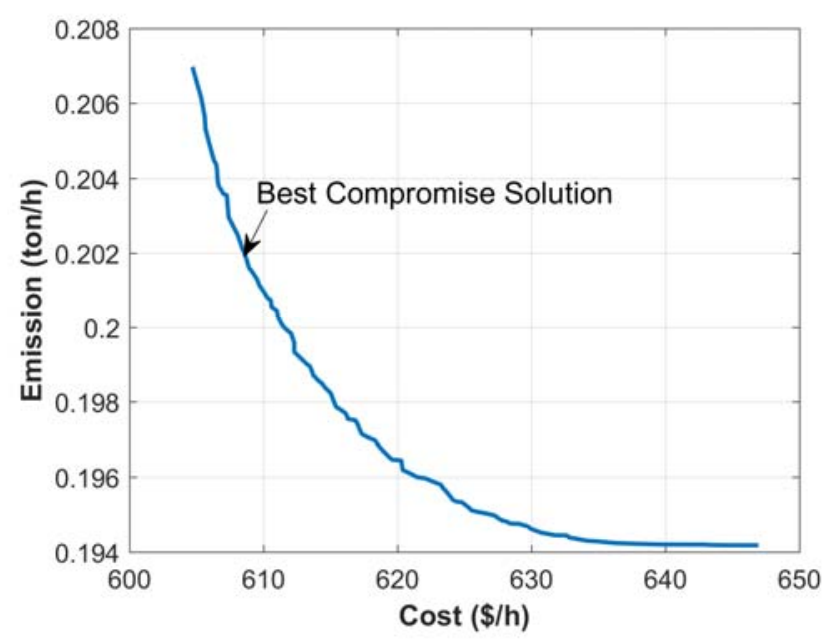

Figure 1. Pareto front produced by IMOPSO for IEEE 30-bus system

Figure 1 demonstrates the Pareto front produced by IMOPSO for the IEEE 30-bus system and shows the relationships between fuel cost and emission. As shown in Table II, the proposed IMOPSO algorithm achieves the best compromise solution with the cost of $607.960 \$ / \mathrm{h}$ and emission of $0.202 \mathrm{ton} / \mathrm{h}$. The fuel cost of IMOPSO is minimum compared to other algorithms; however, the emission is slightly higher than with NPGA [21], SPEA [22] and NSGA [24].

\section{B. Case Study 2}

The second test system is the IEEE 118-bus test system that contains 118-Buses and 14 generators with emission coefficients. The system data of the 118-bus system can be found in the literature [25-26]. The proposed IMOPSO is tested on the IEEE 118 bus, which has a smooth square fuel and emission objective function at a power demand of 950 MW, neglecting transmission losses.

Table III demonstrates the simulation results of IMOPSO, NSGA-III [25] and NSGA-II [27] for the economic dispatch, emission dispatch, and MEED problems. For the 
economic dispatch, the IMOPSO algorithm generates cheaper fuel costs than those of NSGA-III [25] and NSGAII [26]. For the emission dispatch, the IMOPSO algorithm produces a minimum emission compared to other algorithms, as pointed in Table III. Besides, IMOPSO reaches the best compromise solution with a cost of 4330.02 $\$ / \mathrm{h}$ and emission of 123.844 ton $/ \mathrm{h}$ for the MEED problem. The IMOPSO algorithm produces lower fuel costs and minimum emissions compared to NSGA-III [25] and NSGA-II [26].

TABle III. Simulation Results For IEEE 118-Bus Test System

\begin{tabular}{|c|c|c|c|}
\hline Problem & Method & Fuel cost $(\$ / h)$ & $\begin{array}{c}\text { Emission } \\
\text { (ton/h) }\end{array}$ \\
\hline \multirow{3}{*}{ Economic dispatch } & IMOPSO & 4264.51 & 434.154 \\
\hline & NSGA-II [26] & 4358.15 & 290.392 \\
\hline & NSGA-III [25] & 4264.60 & 446.525 \\
\hline \multirow{3}{*}{ Emission dispatch } & IMOPSO & 4511.13 & 17.434 \\
\hline & NSGA-II [26] & 4443.46 & 66.487 \\
\hline & NSGA-III [25] & 4485.00 & 24.093 \\
\hline \multirow{3}{*}{ MEED } & IMOPSO & 4330.02 & 123.844 \\
\hline & NSGA-II [26] & 4391.69 & 129.902 \\
\hline & NSGA-III [25] & 4335.90 & 124.638 \\
\hline \multicolumn{4}{|c|}{ TABLE IV. RESULTS OF IMOPSO FOR MODIFIED IEEE 118-BUS SYSTEM } \\
\hline Problem & Demand (MW) & Fuel cost $(\$ / h)$ & $\begin{array}{c}\text { Emission } \\
\text { (ton/h) }\end{array}$ \\
\hline \multirow{3}{*}{ Economic dispatch } & 950 & 4407.95 & 190.909 \\
\hline & 1500 & 6183.60 & 2002.838 \\
\hline & 2650 & 11315.97 & 7348.820 \\
\hline \multirow{3}{*}{ Emission dispatch } & 950 & 4528.39 & 66.710 \\
\hline & 1500 & 6639.85 & 856.475 \\
\hline & 2650 & 12077.12 & 4893.374 \\
\hline \multirow{3}{*}{ MEED } & 950 & 4495.84 & 77.2831 \\
\hline & 1500 & 6287.06 & 1233.984 \\
\hline & 2650 & 11505.22 & 5501.012 \\
\hline
\end{tabular}

\section{Case Study 3}

The third case study is a modified IEEE 118 bus test system with emission coefficients, ramp rate limits, and prohibited operating zone. Table AI displays the modified IEEE 118 data with ramp rate limits and prohibited zones.

The IMOPSO algorithm is employed to solve economic dispatch, emission dispatch, and MEED problems. Table IV shows the simulation results of IMOPSO for load demands of $950 \mathrm{MW}, 1500 \mathrm{MW}$, and $2650 \mathrm{MW}$. For all demands, the proposed IMOPSO generates minimum costs for the economic dispatch problem and minimum emission amounts for the emission dispatch problem. Furthermore, the IMOPSO algorithm creates the best compromise solutions for solving the MEED problem.

\section{Case Study 4}

In the final case study, the modified IEEE 118 system with 14 conventional units, which is shown in Table AI, is expanded with the addition of six wind farms. The emission coefficients, wind powers, ramp rate limits, and prohibited operating zone are taken into account. Table V shows two different predicted wind speeds of the wind farms. The rated power output $\mathrm{P}_{\mathrm{i}, \mathrm{wr}}$ of each wind generator is considered to be 3 MW. It is assumed that each wind farm consists of 25 identical generators with a total capacity of $75 \mathrm{MW}$. In this study, $v_{r i}=16 \mathrm{~m} / \mathrm{s}, v_{i, i n}=3 \mathrm{~m} / \mathrm{s}$ and $\mathrm{v}_{\mathrm{i}, \text { out }}=25 \mathrm{~m} / \mathrm{s}$ are employed according to the Enercon E82-E4 turbine data [43]. In this paper, the cost coefficient of the wind generator is chosen as 3.25 \$/MW, which is described in [42].

TABLE V. PRedicted Wind SpeEds AND The NumBer OF Wind TURBINES

\begin{tabular}{ccccccc}
\hline Wind Farm & W1 & W2 & W3 & W4 & W5 & W6 \\
$\begin{array}{c}\text { Wind speed 1 } \\
(\mathbf{m} / \mathbf{s})\end{array}$ & 9.3 & 10.5 & 7.6 & 8.2 & 8.7 & 12.6 \\
$\begin{array}{c}\text { Wind speed 2 } \\
(\mathbf{m} / \mathbf{s})\end{array}$ & 10.23 & 11.55 & 8.36 & 9.02 & 9.57 & 13.86 \\
\hline
\end{tabular}

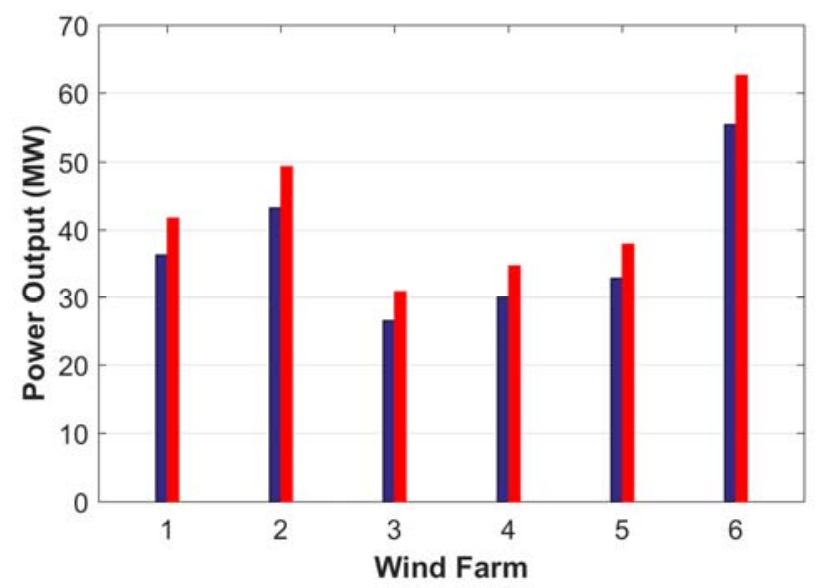

Figure 2. Power outputs of wind farms

TABLE VI. Results For MOdified IEEE 118-Bus Test System With WIND GENERATORS

\begin{tabular}{lcccccc}
\hline & \multicolumn{2}{c}{$\begin{array}{c}\text { Economic } \\
\text { dispatch }\end{array}$} & \multicolumn{2}{c}{$\begin{array}{c}\text { Emission } \\
\text { dispatch }\end{array}$} & \multicolumn{2}{c}{ MEED } \\
\hline Demand (MW) & 1500 & 2650 & 1500 & 2650 & 1500 & 2650 \\
\hline $\begin{array}{l}\text { Wind energy } \\
\text { utilization(MW) }\end{array}$ & 224.423 & 257.25 & 224.423 & 257.25 & 224.423 & 257.25 \\
$\begin{array}{l}\text { Wind power } \\
\text { cost (\$/h) }\end{array}$ & 729.37 & 836.06 & 729.37 & 836.06 & 729.37 & 836.06 \\
$\begin{array}{l}\text { Fuel Cost } \\
\text { \$/h) }\end{array}$ & 5393.13 & 10041.18 & 5748.08 & 10742.795538 .41 & 10259.79 \\
$\begin{array}{l}\text { Total Cost } \\
(\$ / h)\end{array}$ & 6122.50 & 10877.24 & 6477.46 & 11578.856267 .79 & 11095.85 \\
$\begin{array}{l}\text { Emission } \\
\text { (ton/h) }\end{array}$ & 1077.41 & 6023.93 & 428.20 & 3705.40 & 558.26 & 4242.61 \\
\hline
\end{tabular}

Figure 2 shows the power outputs of wind turbines, and the total outputs generated by the wind turbines are 224.423 MW and 257.25 MW respectively. Table VI displays the simulation results of IMOPSO for load demands of 1500 MW and $2650 \mathrm{MW}$. The total production costs of the wind turbines are $729.37 \$ / \mathrm{h}$ and $836.06 \$ / \mathrm{h}$ for the economic dispatch, emission dispatch, and the MEED, as shown in Table VI. The proposed IMOPSO generates minimum costs for the economic dispatch problem and minimum emission amounts for the emission dispatch problem for load demands of $1500 \mathrm{MW}$, and $2650 \mathrm{MW}$.

For the problems of economic dispatch, emission dispatch, and MEED, the total operating costs and emissions presented in Table VI and Table IV are compared with and without wind turbines. For the demand of $1500 \mathrm{MW}$, the total operating costs and emissions for the economic dispatch problem are $6183.60 \$ / \mathrm{h}$ and 2002.838 ton $/ \mathrm{h}$, respectively, while the minimum costs and emissions taking into account the wind turbines, as shown in Table VI, are $6122.50 \$ / \mathrm{h}$ and 1077.415 ton $/ \mathrm{h}$ respectively.

For emission dispatch, the operating costs and emissions are $6639.85 \$ / \mathrm{h}$ and $856.475 \mathrm{t} / \mathrm{h}$, respectively, as indicated in Table IV, while the minimum costs and emissions, 
TABLE A1. Modified IEEE 118 DATA WITH RAMP RATE LIMITS AND PROHIBITED ZONES OF CONVENTIONAL GENERATOR UNITS

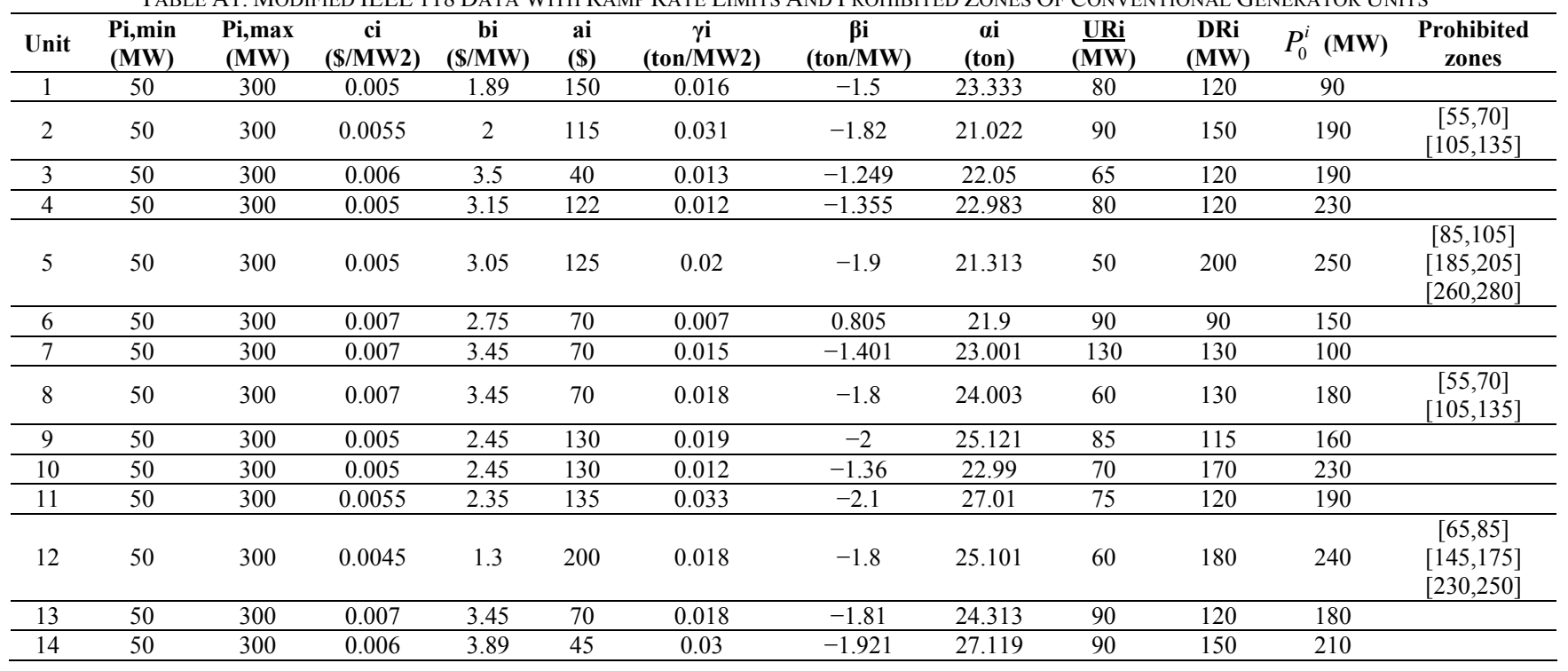

considering wind turbines, are $6477.46 \$ / \mathrm{h}$ and 428.206 ton/h, as shown in Table VI. Finally, the total operating costs and emissions for the MEED problem are $6287.06 \$ / \mathrm{h}$ and 1233.984 ton $/ \mathrm{h}$, respectively, as indicated in Table IV. When wind turbines are included, the minimum costs and emissions are $6267.79 \$ / \mathrm{h}$ and 558.269 ton $/ \mathrm{h}$, respectively, as shown in Table VI.

As seen in Table IV and Table VI, the use of wind turbines significantly reduces total operating costs and pollution emissions at load demands of $1500 \mathrm{MW}$ and 2650 MW for the modified IEEE 118 test system with wind turbines. For economic dispatch, emission dispatch and MEED problems, the emissions are 1077,41 t/h, 428,20 t/h and 558,26 t/h for the $1500 \mathrm{MW}$ demand. The results show that wind power utilization reduces emissions by $46.2 \%$, $50.0 \%$, and $54.7 \%$, respectively, for economic dispatch, emission dispatch, and MEED.

\section{CONCLUSION}

In this paper, the improved multi-objective particle swarm optimization algorithm is presented for the solution of the multi-objective environmental-economic load dispatch problem, taking into account emission pollution, wind power, valve point effects, ramp limits, prohibited operating zones, and transmission losses. The improved multiobjective particle swarm optimization algorithm employs a functional inertia weight and a functional constriction factor to improve the solution. In the IMOPSO algorithm, a mutation strategy is employed, and a mutation operator applied to each particle in the swarm is utilized to find optimal Pareto fronts. The efficiency of IMOPSO was examined with different test systems. The simulation results of the proposed IMOPSO were compared with those of MOGABC [18], MODE/PSO [19], LP [1], MOSST [20], NPGA [21], SPEA [22], FCPSO [23], NSGA [24], NSGAIII [25] and NSGA-II [26]. Compared to other algorithms, IMOPSO can produce fewer fuel costs and emissions by evaluating 2000 fitness function evaluations.

The proposed IMOPSO produces minimum costs for the economic dispatch problem and minimum emission amounts for the emission dispatch problem. Furthermore, the IMOPSO algorithm creates the best compromise solutions for test systems. The simulation results proved that the use of wind turbines considerably reduces the total operating costs and environmental emissions for the economic dispatch, emission dispatch, and MEED problems. The simulation results confirmed that the IMOPSO algorithm represents the useful and potential capability to solve multiobjective EED problems where Pareto optimal solutions are available. For the solution of MEED, the energy storage systems can be addressed in future work.

\section{APPENDIX A}

Table AI shows the modified IEEE 118 data with prohibited zones of conventional generator units.

\section{ACKNOWLEDGMENT}

This work was supported by the Philipp Schwartz Initiative, which was launched by the Alexander von Humboldt Foundation and the German Federal Office.

\section{REFERENCES}

[1] A. Farag, S. Al-Baiyat, T.C. Cheng, "Economic load dispatch multiobjective optimization procedures using linear programming techniques," IEEE Trans. Power Syst., vol.10, pp. 731-738, 1995. doi: $10.1109 / 59.387910$

[2] A. J. Wood, B. F. Wollenberg, G. B. Sheblé, Power Generation, Operation and Control, New Jersey, Wiley-IEEE, 3rd ed., 2013

[3] W. M. Lin, S. J. Chen, "Bid-based dynamic economic dispatch with an efficient interior point algorithm," Int $\mathrm{J}$ of Electrical Power \& Energy Syst., vol. 24, pp. 51-57, 2002. doi:10.1016/S01420615(01)00007-2

[4] L. G. Papageorgiou, E. S. Fraga, "A mixed integer quadratic programming formulation for the economic dispatch of generators with prohibited operating zones," Electr. Power Syst. Res., vol. 77, pp. 1292-1296, 2007. doi:10.1016/j.epsr.2006.09.020

[5] B. Mohammadi-Ivatloo, A. Rabiee and A. Soroudi, "Nonconvex dynamic economic power dispatch problems solution using hybrid immune-genetic algorithm," IEEE Systems Journal, vol. 7, no. 4, pp. 777-785, Dec. 2013, doi:10.1109/JSYST.2013.2258747

[6] Y. Qu, Y. S. Zhu, Y. C. Jiao, et al, "A survey on multi-objective evolutionary algorithms for the solution of the environmental/economic dispatch problems," Swarm and Evolutionary Comp., vol. 38, pp. 1-11, 2018. doi:10.1016/j.swevo.2017.06.002

[7] N. Noman, H. Iba, "Differential evolution for economic load dispatch problems," Elect. Power Syst. Res., vol. 78, pp. 1322-1331, 2008. doi:10.1016/j.epsr.2007.11.007

[8] C. Chelladurai, A. A. Victoire, "Crisscross optimization with comprehensive vertical crossover to solve combined economic 
emission dispatch," Advances in Electrical and Computer Engineering, vol.18, no.3, pp.131-140, 2018 , doi:10.4316/AECE.2018.03018.

[9] A. Bhattacharya, P. K. Chattopadhyay, "Biogeography-based optimization for different economic load dispatch problems," IEEE Trans. Power Syst., vol. 25, pp. 1064-1077, 2010. doi:10.1109/TPWRS.2009.2034525

[10] T. Yalcinoz, K. Rudion, "Economic load dispatch using an improved particle swarm optimization based on functional constriction facto and functional inertia weight," The 19th IEEE Int. Conf. on Environment and Electrical Engineering, Genoa, Italy, 11-14 June 2019. doi:10.1109/EEEIC. 2019.8783432

[11] G. Abbas, J. Gu, U. Farooq, et al, "Solution of an economic dispatch problem through particle swarm optimization: A detailed surveyPart I," IEEE Access, vol. 5, pp. 15105-15141, 2017. doi:10.1109/ACCESS.2017.2723862

[12] V. K. Jadoun, N. Gupta, K. R. Niazi, A. Swarnkar, "Modulated particle swarm optimization for economic emission dispatch," Int J Electr Power Energy Syst., vol. 73, pp. 80-88, 2015. doi:10.1016/j.ijepes.2015.04.004

[13] K. K. Mandal, S. Mandal, B. Bhattacharya, N. Chakraborty, "Nonconvex emission constrained economic dispatch using a new selfadaptive particle swarm optimization technique," Appl. Soft Comput., vol. 28, pp. 188-195, 2015. doi:10.1016/j. asoc.2014.11.033

[14] A. Y. Abdelaziz, E. S. Ali, S. M. Abd Elazim, "Implementation of flower pollination algorithm for solving economic load dispatch and combined economic emission dispatch problems in power systems,' Energy, vol. 101, 506-518, 2016. doi:10.1016/j.energy.2016.02.041

[15] D. C. Secui, "A method based on the ant colony optimization algorithm for dynamic economic dispatch with valve point effects," Int Trans Electr Energ Syst., vol. 25, pp. 262-287, 2015. doi:10.1002/etep. 1841

[16] S. Hemamalini, S. P. Simon, "Dynamic economic dispatch using artificial immune system for units with valve-point effect," Int J Electr Power Energy Syst., vol. 33, pp. 868-874, 2011. doi:10.1016/j.ijepes.2010.12.017

[17] I. A. Farhat, M. E. El-Hawary, "Dynamic adaptive bacterial foraging algorithm for optimum economic dispatch with valve-point effects and wind power," IET Gener Trans Distrib., vol. 4, pp. 989-999, 2010. doi:10.1049/iet-gtd.2010.0109

[18] S. Ma, Y. Wang, Y. Lv, "Multiobjective environment/ economic power dispatch using evolutionary multiobjective optimization," IEEE Access, vol. 6, pp. 13066-13074, 2018 doi:10.1109/ACCESS.2018.2795702

[19] D. W. Gong, Y. Zhang, C. L. Qi, "Environmental/economic power dispatch using a hybrid multi-objective optimization algorithm," Int J. Electr Power Energy Syst., vol. 32, pp. 607-614, 2010 doi:10.1016/j.ijepes.2009.11.017

[20] D. B. Das, C. Patvardhan, "New multi-objective stochastic search technique for economic load dispatch," IEE Proc Gener Trans Distrib., vol. 145, pp. 747-752, 1998. doi:10.1049/ip-gtd:19982367

[21] M. A. Abido, "A niched Pareto genetic algorithm for multiobjective environmental/ economic dispatch," Int J Electr Power Energy Syst., vol. 25, pp. 97-105, 2003. doi:10.1016/S0142-0615(02)00027-3

[22] M. A. Abido, "Multiobjective evolutionary algorithms for electric power dispatch problem," IEEE Trans Evol Comput., vol. 10, pp. 315-329, 2006. doi:10.1109/TEVC.2005.857073

[23] S. Agrawal, B. K. Panigrahi, M. K. Tiwari, "Multiobjective particle swarm algorithm with fuzzy clustering for electrical power dispatch," IEEE Trans Evol Comput., vol. 12, pp. 529-541, 2008 doi:10.1109/TEVC.2007.913121

[24] M. A. Abido, "A novel multiobjective evolutionary algorithm for environmental/economic power dispatch," Electr Power Syst Res. vol. 65, pp. 71-81, 2003. doi:10.1016/S0378-7796(02)00221-3

[25] R. H. Bhesdadiya, I. N. Trivedi, P. Jangir, et al, "An NSGA-III algorithm for solving multi-objective economic/environmental dispatch problem," Cogent Eng., vol. 3, pp. 1269383, 2016. doi: $10.1080 / 23311916.2016 .1269383$
[26] S. Sivasubramani, K. S. Swarup, "Environmental/economic dispatch using multi-objective harmony search algorithm," Electr Power Syst Res., vol. 81, pp. 1778-1785, 2011. doi:10.1016/j.epsr.2011.04.007

[27] J. Kennedy, R.C. Eberhart, "Particle swarm optimization," Proceedings of ICNN'95 - International Conference on Neura Networks Perth, WA, Australia, pp. 1942-1948, 1995. doi:10.1109/ICNN.1995.488968

[28] M. R. Bonyadi, Z. Michalewicz, "Particle swarm optimization for single objective continuous space problems: a review," Evolutionary Computation, vol. 25, pp. 1-54, 2017. doi:10.1162/EVCO_r_00180

[29] M. Clerc, J. Kennedy, "The particle swarm explosion, stability, and convergence in a multidimensional complex space," IEEE Trans Evolutionary Computation," vol. 6, pp.58-73, 2002 doi:10.1109/4235.985692

[30] A. Ratnaweera, S. K. Halgamuge, H. C. Watson, "Self-organizing hierarchical particle swarm optimizer with time-varying acceleration coefficients," IEEE Trans Evolutionary Computation, vol. 8, pp. 240 255, 2004. doi:10.1109/tevc.2004.826071

[31] C. Sun, J. Zeng, J. Pan, S. Xue, Y. Jin, "A new fitness estimation strategy for particle swarm optimization," Information Sciences," vol. 221, pp. 355-370, 2013. doi:10.1016/j.ins.2012.09.030

[32] A. Nickabadi, M. M. Ebadzadeh, R. Safabakhsh, "A novel particle swarm optimization algorithm with adaptive inertia weight," Applied Soft Comp., vol. 11, pp. 3658-3670, 2011 doi:10.1016/j.asoc.2011.01.037

[33] Y. Lu, M. Liang, Z. Ye, L. Cao, "Improved particle swarm optimization algorithm and its application in text feature selection," Applied Soft Comp., vol. 35, pp. 629-636, 2015 doi:10.1016/j.asoc.2015.07.005

[34] M. Abido, "Optimal power flow using particle swarm optimization," Int J Electr Power Energy Syst., vol. 24, pp. 563-571, 2002. doi:10.1016/S0142-0615(01)00067-9

[35] J. G. Vlachogiannis, K. Y. Lee, "A comparative study on particle swarm optimization for optimal steady-state performance of power systems," IEEE Trans. Power Syst., vol. 21, pp. 1718-1727, 2006. doi:10.1109/TPWRS.2006.883687

[36] G. Pranava, P.V. Prasad, "Constriction coefficient particle swarm optimization for economic load dispatch with valve point loading effects," Int Conf Power Energy Control, Sri Rangalatchum Dindigul, India, pp. 350-354, 2013. doi:10.1109/ICPEC.2013.6527680

[37] C. C. A. Coello, G. T. Pulido, M. S. Lechuga, "Handling multiple objectives with particle swarm optimization," IEEE Trans Evol Comput., vol. 8, pp. 256-279, 2004. doi:10.1109/tevc.2004.826067

[38] C. C. A. Coello, "An introduction to multi-objective particle swarm optimizers," Gaspar-Cunha A, Takahashi R, Schaefer G, Costa L, (eds) Soft Computing in Industrial Applications, Advances in Intelligent and Soft Computing, Springer, Berlin, Heidelberg, pp. 96 , 2011. doi:https://doi.org/10.1007/978-3-642-20505-7_1

[39] P. K. Tripathi, S. Bandyopadhyay, S. K. Pal, "Multi-objective particle swarm optimization with time variant inertia and acceleration coefficients," Information Sciences, vol. 177, pp. 5033-5049, 2007. doi:10.1016/j.ins.2007.06.018

[40] O. R. Castro, G. M. Fritsche, A. Pozo, "Evaluating selection methods on hyper-heuristic multi-objective particle swarm optimization," Heuristics, vol. 24, pp. 581-616, 2018. doi:10.1007/s10732-0189369-X

[41] C. L. Chen, T. Y. Lee, R. M. Jan, "Optimal wind-thermal coordination dispatch in isolated power systems with large integration of wind capacity," Energy Conversion and Management, vol. 47, pp. 3456-3472, 2006. doi:10.1016/j.enconman.2005.12.016

[42] S. S. Reddy, "Optimal scheduling of thermal-wind-solar power system with storage," Renewable Energy, vol. 101, pp. 1357-1368, 2017. doi:10.1016/j.renene.2016.10.022

[43] P. Partha, P. N. Biswas, B. Y. Suganthan, et al, "Multiobjective economic-environmental power dispatch with stochastic wind-solarsmall hydro power," Energy, vol. 150, pp. 1039-1057, 2018 doi:10.1016/j.energy.2018.03.002 